Rapid Reviews COVID-19

\title{
Review 1: "COVID-19 and its clinical severity are associated with alterations of plasma sphingolipids and enzyme activities of sphingomyelinase and ceramidase"
}

Johnny Stiban ${ }^{1}$

${ }^{1}$ Birzeit University

Published on: Mar 01, 2022

License: Creative Commons Attribution 4.0 International License (CC-BY 4.0). 


\section{$\underline{\text { RR:C19 Evidence Scale rating by reviewer: }}$}

- Potentially informative. The main claims made are not strongly justified by the methods and data, but may yield some insight. The results and conclusions of the study may resemble those from the hypothetical ideal study, but there is substantial room for doubt. Decision-makers should consider this evidence only with a thorough understanding of its weaknesses, alongside other evidence and theory. Decisionmakers should not consider this actionable, unless the weaknesses are clearly understood and there is other theory and evidence to further support it.

******************************************

\section{Review:}

This manuscript entitled "COVID-19 and its clinical severity is associated with alterations of plasma sphingolipids and enzyme activities of sphingomyelinase and ceramidase" provides timely and important research that links COVID-19 infection with plasma sphingolipid metabolism. The authors investigated plasma activities of sphingomyelinases and ceramides in a large patient cohort diagnosed with or recovering from COVID-19. The study concludes that acid and neutral sphingomyelinases showed increased activities whereas the activity neutral ceramidase was reduced in COVID-19 patients. These changes lead to increased plasma ceramide levels in patients and the study postulates that this increase enhances the infectability of the coronavirus. The study shows a COVID-19-dependent alteration of sphingolipid homeostasis that is dependent on the severity of the symptoms. The authors postulate that using approved drugs that counter the effects of increased ceramides and decreased sphingomyelins would be effective to combat COVID-19, although without presenting evidence for this claim.

This report can be addressed as a preliminary work that requires further validation and more mechanistic approach to become suitable for publication. The data they present is compelling in terms of increased ceramide levels and modulation of two key enzymes in the sphingolipid metabolic pathway. However, that is only what the report shows and other insinuations about FIASMA and potential therapies are speculations and not supported by actual data. That being said, I commend the authors for their work but I advise them to revise the discussion and conclusion to remove the potential 
therapeutic outcome, or to include data from FIASMA-treated COVID-19 patients to support their claims.

According to the journal's guidelines, I would rate this manuscript as potentially informative, since the data from sphingomyelinase and ceramidase and the concomitant lipid species analysis are compelling and statistically significant. However, other sphingolipid metabolizing enzymes need to be explored, especially enzymes in the de novo synthesis. Also, there is no presented evidence that using FIASMA will lead to alleviation ofCOVID-19 symptoms and hospitalization. Moreover, the report measures ceramides and enzyme activities in plasma and serum, but does not address tissue ceramide levels or activities. There is also no mention of apoptosis and other ceramide-dependent physiological and pathophysiological pathways that are affected by the accumulation of ceramides. I should also mention that the authors failed to cite a very relevant recent review on sphingolipids as disease biomarkers (PMID: 31502202).

As a summary, I think the report is important and timely but it is stretching it a little bit. I would accept it if the authors restructure it to give lower importance for FIASMA and potential therapies and focus on the significant effect they saw in ceramide accumulation by sphingomyelinase activation and ceramidase inhibition.

- Minor comments:

- I think there is a mistake in the discussion when they refer to acid and neutral ceramidase (AN/NC). I believe it should be (AC/NC).

- I wonder why not all authors' contributions are listed, as only a handful of authors had descriptions of their endeavors. 